\title{
ESTIMATES FOR A CLASS OF SLOWLY NON-DISSIPATIVE REACTION-DIFFUSION EQUATIONS
}

\author{
EDGARD A. PIMENTEL AND JULIANA F.S. PIMENTEL
}

\begin{abstract}
In this paper, we consider slowly nondissipative reaction-diffusion equations and establish several estimates. In particular, we manage to control $L^{p}$ norms of the solution in terms of $W^{1,2}$ norms of the initial conditions, for every $p>2$. This is done by carefully combining preliminary estimates with Gronwall's inequality and the Gagliardo-Nirenberg interpolation theorem. By considering only positive solutions, we obtain upper bounds for the $L^{p}$ norms, for every $p>1$, in terms of the initial data. In addition, explicit estimates concerning perturbations of the initial conditions are established. The stationary problem is also investigated. We prove that $L^{2}$ regularity implies $L^{p}$ regularity in this setting, while further hypotheses yield additional estimates for the bounded equilibria. We close the paper with a discussion of the connection between our results and some related problems in the theory of slowly non-dissipative equations and attracting inertial manifolds.
\end{abstract}

1. Introduction. The study of reaction-diffusion equations has been mainly motivated by their large range of applications, appearing in interaction models in a variety of contexts. Examples are to be found, for instance, in population dynamics, chemical reactions, as well as in the realm of biology or physics. Dissipative reactiondiffusion equations have been investigated by numerous authors and various problems have already been worked out in detail. For general dissipative settings, see $[\mathbf{3}, \mathbf{1 4}, \mathbf{1 6}, \mathbf{1 7}]$. For scalar reaction-diffusion equations, see $[\mathbf{8}, \mathbf{9}, \mathbf{1 1}, \mathbf{2 0}]$. Likewise, fast non-dissipative equations, i.e., those exhibiting blow-up in finite time, have also been extensively studied in the literature.

2010 AMS Mathematics subject classification. Primary 35B65, 35K57, 58J35.

Keywords and phrases. Slowly non-dissipative systems, reaction-diffusion equations, Gagliardo-Nirenberg inequality, Sobolev regularity.

The first author was supported by $\mathrm{CNPq}-$ Brazil. The second author was supported by CNPq-Brazil and FAPESP.

Received by the editors on March 11, 2014 and in revised form on June 29, 2014. 
Despite the scientific developments regarding dissipative, as well as fast non-dissipative equations, the class of scalar reaction-diffusion equations with global existence and a set of solutions blowing-up in infinite time has not been approached until recently. These were introduced in [6]. This class of dynamical systems is referred to as slowly non-dissipative systems (SND). In other words, the dynamical system generated by a scalar reaction-diffusion equation is said to be slowly non-dissipative if global well-posedness is ensured but at least one solution grows up to infinity with time. Such an unbounded solution is referred to as a grow-up solution.

In recent years, a qualitative theory of the asymptotic behavior of these systems has been established $[\mathbf{4}, \mathbf{5}, \mathbf{6}, \mathbf{1 8}, \mathbf{1 9}]$. However, to the best of our knowledge, there is no information regarding the quantitative behavior of slowly non-dissipative dynamical systems.

In this paper, we consider the following model SND equation:

$$
\begin{cases}u_{t}-u_{x x}=b u+g\left(x, u, u_{x}\right) & \text { in }[0, \pi] \times(0,+\infty) \\ u(x, 0)=u_{0}(x) & \text { on }[0, \pi] \times\{t=0\},\end{cases}
$$

where $b>0$ is a scalar, $u_{0}:[0, \pi] \rightarrow \mathbb{R}$ is a function in the appropriate state space and $g$ satisfies certain conditions, to be specified later. We equip (1.1) with the so-called homogeneous Neumann boundary conditions, i.e.,

$$
u_{x}(0, t)=u_{x}(\pi, t)=0
$$

for all $t>0$.

In the present paper, estimates for the solutions of (1.1)-(1.2) are obtained under fairly natural assumptions on $b$ and $g$, see Section 2 . At first, we control norms of $u$ in $L^{p}$ spaces in terms of $W^{1,2}$ norms of the initial condition, for every $p>2$. This is done by carefully combining Gronwall's inequality and ideas inspired by the adjoint method, see [10], with the Gagliardo-Nirenberg interpolation theorem. Our result yields an explicit estimate for the norms of the solutions in $L^{p}$ spaces, in terms of the nonlinearity and the initial data. This provides an exponential growth rate for the solutions, as $t \rightarrow \infty$. In particular, this upper bound improves and extends previous results obtained in [21]. By considering only positive solutions, we also obtain an estimate for the $L^{p}$-norms of $u$, for $p>1$. 
As an auxiliary result, we establish $L^{2}$ estimates for solutions of (1.1)-(1.2) in terms of $L^{2}$-norms of the initial data. This class of estimates is relevant for it provides a better estimate than that obtained in $[\mathbf{1 8}]$ for $u(\cdot, t)$, for each time $t>0$, in fractional power spaces strictly contained in $L^{2}$. See Remark 5.4.

Moreover, we study the dependence of the solutions with respect to perturbations of the initial conditions. Our findings show that, for every arbitrarily fixed $t>0$, solutions starting $\varepsilon$-close to each other remain $e^{C t} \varepsilon$-close, where $C>0$, depending on $b$ and $g$, is explicitly determined. This result improves the estimate for the norms of the solutions both in $L^{p}$, for $p \geq 2$, and in $W^{1,2}$.

Lastly, we investigate the associated stationary system. In this setting, we show that regularity in $L^{2}$ implies regularity in $L^{p}$, for $2<p \leq \infty$. This result follows from the Gagliardo-Nirenberg theorem, combined with a series of estimates and Gronwall's inequality. By imposing further conditions on the parameter $b$ we also manage to obtain a uniform estimate for the bounded equilibria, depending solely on $b$ and $g$.

Although the relevance of this class of results is justified on its own mathematical merit, the motivation for the study of quantitative properties of solutions to (1.1) is twofold.

On one hand, it is essential to understand how the different norms of the grow-up solutions depend on known quantities of the problem, e.g., the nonlinearity $g$ as well as the term $b$ and the initial data. For instance, it is reasonable to expect that a grow-up solution of (1.1)-(1.2) satisfies an exponential growth condition depending on $b$, see $[\mathbf{5}, \mathbf{1 8}]$. Our results provide a rigorous proof of this fact, accounting also for the dependence on the nonlinearity $g$.

Furthermore, the findings in this paper enable us to recover certain qualitative properties of the associated dynamical system, since our estimates reflect results previously obtained in the literature. For example, because some upper bounds in Section 6 depend explicitly on $b$, limits can be computed by simply letting $b \rightarrow 0$ or $b \rightarrow 1$. In this concrete case, our results are consistent with the qualitative theory for $(1.1)-(1.2)$. See $[\mathbf{5}, \mathbf{1 5}]$. In addition, our estimates regarding the dependence on the initial data yield an upper bound for the grow- 
up solutions, which compare with results on the theory of inertial manifolds, see [13].

Despite the fact that the results in this paper regard the scalar case, the techniques presented do not further rely on the dimension. It indicates that our findings can add to the quantitative theory of reaction-diffusion equations in higher dimensions as well. Moreover, the technical arguments used in this paper, and the delicate ways in which they can be combined, may yield various estimates for (coupled) non-linear partial differential equations. See, for example, the recent results obtained in [12].

The remainder of this paper is organized as follows. Section 2 presents the main assumptions used throughout the paper. For the sake of completeness, we provide a brief discussion on slowly nondissipative systems in Section 3. In Section 4, we establish $L^{p}$ regularity for the solutions of (1.1)-(1.2). An explicit estimate for the dependence of solutions with respect to perturbations of the initial conditions is provided in Section 5. Also, improvements of $L^{p}$ estimates are provided. The stationary problem is dealt with in Section 6. In this section, we show that $L^{2}$ regularity implies $L^{p}$ regularity. By imposing a further constraint on $b$, we also establish a uniform bound for the $L^{2}$ norms of the solution in terms of a constant depending only on $b$ and $g$. This builds upon the previous result to ensure uniform bounds for the equilibria of the system in $L^{p}$. A concluding section summarizes our findings and highlights their relevance in the context of reactiondiffusion equations.

2. Main assumptions. In what follows, we state the main assumptions under which we work throughout the paper.

Assumption A1. We assume that $u_{0} \in X^{\alpha} \subset \mathcal{C}^{1}([0, \pi])$.

In Assumption A1, $X^{\alpha}$ is a fractional power space to be defined later, see Section 3. The next group of assumptions regards the nonlinearity, $g$.

Assumption A 2. We assume $g \in \mathcal{C}^{2}\left([0, \pi] \times \mathbb{R}^{2}\right)$, and there exists $C^{g}>0$ such that

$$
|g(x, u, p)| \leq C^{g}
$$


and

$$
|g(x, u, p)-g(x, v, q)| \leq C^{g}(|u-v|+|p-q|),
$$

for all $(x, u, p),(x, v, q) \in[0, \pi] \times \mathbb{R}^{2}$.

Assumption A2 is critical also for the global well-posedness of solutions to (1.1)-(1.2), see [16].

Assumption A3. We assume $g=g(u)$. Furthermore, $g \in \mathcal{C}^{2}(\mathbb{R})$, and there exists $C^{g}>0$ such that

$$
|g(u)|,\left|g^{\prime}(u)\right| \leq C^{g},
$$

for all $u \in \mathbb{R}$.

The next assumption regards the linear coefficient $b$.

Assumption A4. We assume that $0<b<1$.

The next section presents a brief discussion on slowly non-dissipative dynamical systems.

3. Slowly non-dissipative equations. It is known that the dynamical system induced by equation (1.1) is slowly non-dissipative. That is to say, global existence and uniqueness are guaranteed for all initial conditions $u_{0}$ in the state space, but a subset of solutions exhibits blow-up in infinite time. These will be verified within this section.

In the case of equation $(1.1)$, the $L^{2}([0, \pi])$ space as the underlying space would not provide enough regularity. Then, to overcome this fact, we consider fractional power spaces of $X=L^{2}([0, \pi])$. We consider the operator $A=-\partial_{x x}-b I . \quad A$ is a sectorial operator in $X$ and $A_{1}=A+(b+1) I$ is a positive operator. These imply that the fractional power spaces

$$
X^{\alpha} \doteq D\left(A_{1}^{\alpha}\right), \quad \alpha \geq 0,
$$

are well defined with the graph norm $\|x\|_{\alpha}=\left\|A_{1}^{\alpha} x\right\|$, where $\|\cdot\|$ denotes the usual norm in $L^{2}([0, \pi])$. We take $X^{\alpha}$ with $\alpha>3 / 4$ to be the underlying space, since we have from embedding theory that in this case $X^{\alpha}$ is contained in $C^{1}([0, \pi])$; see, for example, [16]. 
From the conditions we have imposed on the nonlinearity $g$ and from the standard semigroup theory $[\mathbf{2}, \mathbf{1 4}, \mathbf{1 6}]$ we can ensure local existence and uniqueness for any solution $u(\cdot, t)$ of $(1.1)-(1.2)$. Therefore, the associated semigroup $S_{t}$ is defined in $X^{\alpha}$, for $t \geq 0$,

$$
\begin{aligned}
S_{t}: X^{\alpha} & \longrightarrow X^{\alpha}, \\
u_{0} & \longmapsto u\left(\cdot, t ; u_{0}\right),
\end{aligned}
$$

where $u\left(\cdot, t ; u_{0}\right)$ denotes the solution of (1.1)-(1.2). Moreover, we know from [16] that either the maximal time of existence $t_{u_{0}}$ of the solution $u\left(\cdot, t ; u_{0}\right)$ is equal to infinity or

$$
\lim _{t \rightarrow t_{u_{0}}}\left\|S_{t}\left(u_{0}\right)\right\|_{\alpha}=\infty
$$

If we set $G(u)(x) \doteq g\left(x, u, u_{x}\right)$, then $g$ being bounded implies that $\|G(u)\| \leq M$ for some positive constant $M \in \mathbb{R}$. We thus have the following, for any solution $u(\cdot, t)$ of $(1.1)-(1.2)$ and $3 / 4<\alpha<1$,

$$
\begin{aligned}
\|u(\cdot, t)\|_{\alpha} & =\left\|S_{t}\left(u_{0}\right)\right\|_{\alpha} \\
& =\left\|e^{-A t} u_{0}+\int_{0}^{t} e^{-A(t-s)} G(u) d s\right\|_{\alpha} \\
& \leq C e^{b t}\left\|u_{0}\right\|_{\alpha}+M \int_{0}^{t}(t-s)^{-\alpha} e^{b(t-s)} d s,
\end{aligned}
$$

which is bounded for each $0<t<\infty$, see [18]. This implies global existence for all the initial conditions $u_{0} \in X^{\alpha}$.

To conclude that the induced dynamical system $S_{t}$ is slowly nondissipative it remains to obtain the existence of a subset of unbounded solutions. For that, we refer the reader to [6], where the author obtains the existence of a solution blowing-up in infinite time just by assuming that $b>0$.

The existence of a non-compact global attractor associated with the dynamical system $S_{t}$ is obtained in $[\mathbf{6}, \mathbf{1 8}, \mathbf{1 9}]$. This object is defined to be the non-empty minimal set in $X^{\alpha}$ attracting all bounded subsets of $X^{\alpha}$. The existence of unbounded trajectories, i.e., grow-up solutions, requires the introduction of some objects at infinity that are interpreted as equilibria at infinity. It is obtained in $[6, \mathbf{1 8}, \mathbf{1 9}]$ that the noncompact global attractor is entirely composed by bounded equilibrium points, equilibria at infinity and heteroclinic orbits connecting them. 
In the next section, we obtain $L^{p}$ estimates for a solution of (1.1)(1.2) in terms of norms of the initial condition in Sobolev spaces.

4. Estimates in $L^{p}$ spaces. We start by investigating $L^{2}$ estimates for $u$.

Proposition 4.1 ( $L^{2}$-regularity). Let $u$ be a solution to (1.1)-(1.2), and assume that Assumptions $\mathrm{A} 1$ and $\mathrm{A} 2$ hold. Then:

$$
\|u(\cdot, t)\|_{L^{2}([0, \pi])}^{2} \leq e^{2(b+\varepsilon) t}\left(C_{t, g, \varepsilon}+\left\|u_{0}\right\|_{L^{2}([0, \pi])}^{2}\right),
$$

where the constant $C_{t, g, \varepsilon}$ is given by

$$
C_{t, g, \varepsilon} \equiv \frac{t \pi}{2 \varepsilon}\left(C^{g}\right)^{2}
$$

with $\varepsilon>0$.

Proof. Multiply (1.1) by $u$ and integrate by parts to obtain

(4.1) $\frac{1}{2} \frac{d}{d t} \int_{0}^{\pi} u^{2}(x, t) d x \leq b \int_{0}^{\pi} u^{2}(x, t) d x+\int_{0}^{\pi} g\left(x, u, u_{x}\right) u(x, t) d x$.

By integrating $(4.1)$ on $(0, \tau)$ with respect to the Lebesgue measure, and noticing that

$$
\left|g\left(x, u, u_{x}\right) u(x, t)\right| \leq \varepsilon u^{2}+\frac{g^{2}\left(x, u, u_{x}\right)}{4 \varepsilon},
$$

it follows that

$$
\begin{aligned}
\int_{0}^{\pi} u^{2}(x, \tau) d x \leq & 2(b+\varepsilon) \int_{0}^{\tau} \int_{0}^{\pi} u^{2}(x, t) d x d t \\
& +\frac{2}{4 \varepsilon} \int_{0}^{\tau} \int_{0}^{\pi} g^{2}\left(x, u, u_{x}\right) d x d t+\int_{0}^{\pi} u_{0}^{2}(x) d x \\
\leq & 2(b+\varepsilon) \int_{0}^{\tau} \int_{0}^{\pi} u^{2}(x, t) d x d t \\
& +\frac{\tau \pi}{2 \varepsilon}\left(C^{g}\right)^{2}+\int_{0}^{\pi} u_{0}^{2}(x) d x
\end{aligned}
$$


Gronwall's inequality then yields

$$
\|u(\cdot, t)\|_{L^{2}([0, \pi])}^{2} \leq e^{2(b+\varepsilon) t}\left(\frac{t \pi}{2 \varepsilon}\left(C^{g}\right)^{2}+\left\|u_{0}\right\|_{L^{2}([0, \pi])}^{2}\right),
$$

which concludes the proof.

Next, we obtain bounds in $L^{\infty}$ for the solution $u$.

Proposition 4.2 ( $L^{\infty}$-regularity). Let $u$ be a solution to (1.1)-(1.2), and assume that Assumptions A1-A2 hold. Then,

$$
\|u(\cdot, t)\|_{L^{\infty}([0, \pi])} \leq e^{b t}\left(t C^{g}+\left\|u_{0}\right\|_{L^{\infty}([0, \pi])}\right),
$$

for every $t>0$.

Proof. Consider the backwards heat equation

$$
\begin{cases}\rho_{t}+\rho_{x x}=0 & \text { in }[0, \pi] \times(0, \tau) \\ \rho(x, \tau)=\delta_{x_{0}}(x) & \text { on }[0, \pi] \times\{t=\tau\},\end{cases}
$$

where $\delta_{x_{0}}$ is the Dirac delta centered at $x_{0} \in(0, \pi)$. We equip (4.2) with homogeneous Neumann boundary conditions. It is clear that $\rho$ is the density of a probability measure for all times $t \in[0, \tau]$.

Multiply (1.1) by $\rho$ and (4.2) by $u$, sum and integrate by parts to obtain:

$$
\begin{aligned}
\left|u\left(x_{0}, \tau\right)\right| \leq & b \int_{0}^{\tau} \int_{0}^{\pi}|u(x, t)| \rho(x, t) d x d t \\
& +\int_{0}^{\tau} \int_{0}^{\pi}\left|g\left(x, u, u_{x}\right)\right| \rho(x, t) d x d t \\
& +\int_{0}^{\pi}\left|u_{0}(x)\right| \rho(x, 0) d x .
\end{aligned}
$$

Therefore,

$$
\sup _{x}|u(x, \tau)| \leq b \int_{0}^{\tau} \sup _{x}|u(x, t)| d t+\tau C^{g}+\left\|u_{0}\right\|_{L^{\infty}([0, \pi])},
$$

since $x_{0}$ is arbitrarily chosen. Gronwall's inequality then yields the result.

In what follows, we obtain an estimate for the $L^{2}$-norm of $u_{x}$. 
Proposition 4.3 (Gradient $L^{2}$-regularity). Let $u$ be a solution to (1.1)-(1.2), and assume that Assumptions A1 and A3 hold. Then,

$$
\left\|u_{x}(\cdot, t)\right\|_{L^{2}([0, \pi])}^{2} \leq e^{2\left(b+C^{g}\right) t}\left\|\left(u_{0}\right)_{x}\right\|_{L^{2}([0, \pi])}^{2},
$$

for every $t>0$.

Proof. Differentiate (1.1) with respect to $x$ and multiply it by $u_{x}$. Integrate by parts to obtain

$$
\frac{d}{d t} \int_{0}^{\pi} \frac{u_{x}^{2}(x, t)}{2} d x=-\int_{0}^{\pi} u_{x x}^{2} d x+\int_{0}^{\pi}\left(b+g^{\prime}(u)\right) u_{x}^{2}(x, t) d x .
$$

Hence,

$$
\int_{0}^{\pi} u_{x}^{2}(x, \tau) d x \leq 2\left(b+C^{g}\right) \int_{0}^{\tau} \int_{0}^{\pi} u_{x}^{2}(x, t) d x+\int_{0}^{\pi}\left(u_{0}\right)_{x}^{2}(x) d x .
$$

A further application of Gronwall's inequality yields the result.

Corollary 4.4 (Sobolev regularity). Let $u$ be a solution to (1.1)-(1.2), and assume that Assumptions A1 and A3 hold. Then, for $\varepsilon>0$,

$$
\|u(\cdot, t)\|_{W^{1,2}([0, \pi])}^{2} \leq C_{t, g, \varepsilon} e^{2(b+\varepsilon) t}+e^{2\left(b+C^{g}\right) t}\left\|u_{0}\right\|_{W^{1,2}([0, \pi])}^{2},
$$

for every $t>0$, where $C_{t, g, \varepsilon}$ depends only on $t, g$ and $\varepsilon$.

Proof. By combining Propositions 4.1 and 4.3, we have $\|u(\cdot, t)\|_{W^{1,2}([0, \pi])} \leq e^{2(b+\varepsilon) t}\left(C_{t, g, \varepsilon}+\left\|u_{0}\right\|_{L^{2}([0, \pi])}^{2}\right)+e^{2\left(b+C^{g}\right) t}\left\|\left(u_{0}\right)_{x}\right\|_{L^{2}([0, \pi])}^{2}$.

Because $\varepsilon$ can be taken such that $\varepsilon \leq C^{g}$, it follows that

$$
\|u(\cdot, t)\|_{W^{1,2}([0, \pi])} \leq C_{t, g, \varepsilon} e^{2(b+\varepsilon) t}+e^{2\left(b+C^{g}\right) t}\left\|u_{0}\right\|_{W^{1,2}([0, \pi])}^{2} .
$$

Corollary 4.4 is then established.

Proposition 4.5. Let $u$ be a solution to (1.1)-(1.2), and assume that Assumptions A1 and A3 hold. Then there exists a constant $C_{t, g}>0$, depending solely on $t$ and $g$, such that

$$
\|u(\cdot, t)\|_{L^{p}([0, \pi])} \leq C_{t, g} e^{\left(b+C^{g}\right) t}\left(1+\left\|u_{0}\right\|_{W^{1,2}([0, \pi])}\right),
$$

for every $p>2$ and $t>0$. 
Proof. The Gagliardo-Nirenberg inequality implies

$$
\|u(\cdot, t)\|_{L^{p}([0, \pi])} \leq C\|u(\cdot, t)\|_{L^{2}([0, \pi])}^{1-\alpha}\|u(\cdot, t)\|_{W^{1,2}([0, \pi])}^{\alpha},
$$

where

$$
\alpha=\frac{p-2}{2 p} .
$$

See [7]. By referring to (4.3) and combining Proposition 4.1 and Corollary 4.4, one has

$$
\begin{aligned}
\|u(\cdot, t)\|_{L^{p}([0, \pi])} \leq & C\left[e^{\left(b+C^{g}\right) t}\left(C_{t, g}+\left\|u_{0}\right\|_{L^{2}([0, \pi])}\right)\right]^{1-\alpha} \\
& \times\left[e^{\left(b+C^{g}\right) t}\left(C_{t, g}+\left\|u_{0}\right\|_{W^{1,2}([0, \pi])}\right)\right]^{\alpha},
\end{aligned}
$$

provided we take $\varepsilon \equiv C^{g}$. Therefore,

$$
\begin{aligned}
\|u(\cdot, t)\|_{L^{p}([0, \pi])} & \leq C_{t, g} e^{\left(b+C^{g}\right) t}\left(1+\left\|u_{0}\right\|_{L^{2}([0, \pi])}^{1-\alpha}+\left\|u_{0}\right\|_{W^{1,2}([0, \pi])}^{\alpha}\right) \\
& \leq C_{t, g} e^{\left(b+C^{g}\right) t}\left(1+\left\|u_{0}\right\|_{W^{1,2}([0, \pi])}+\left\|u_{0}\right\|_{W^{1,2}([0, \pi])}\right),
\end{aligned}
$$

where we have used elementary facts about norms in Sobolev spaces combined with Young's inequality.

To close this section, we consider positive solutions of (1.1)-(1.2). This is motivated by problems involving positive quantities, e.g., densities.

Proposition 4.6. Let $u$ be a positive solution to (1.1)-(1.2), and assume that Assumptions A1-A2 hold. Then,

$$
\|u(\cdot, t)\|_{L^{p}([0, \pi])}^{p} \leq e^{\left(b p^{2}+p-1 / p\right) t}\left(\left\|u_{0}\right\|_{L^{p}([0, \pi])}^{p}+\left(C^{g}\right)^{p} t\right),
$$

for every $1<p<\infty$.

Proof. Multiply (1.1) by $p u^{p-1}$ and integrate by parts to obtain

$$
\begin{aligned}
& \frac{d}{d t} \int_{0}^{\pi} u^{p}(x, t) d x=\int_{0}^{\pi} b p u^{p}(x, t) d x \\
& \quad+p \int_{0}^{\pi} g\left(x, u, u_{x}\right) u^{p-1}(x, t)-\frac{4(p-1)}{p} \int_{0}^{\pi}\left|u_{x}^{p / 2}(x, t)\right|^{2} d x .
\end{aligned}
$$


Young's inequality implies that

$$
\begin{aligned}
\frac{d}{d t} \int_{0}^{\pi} u^{p}(x, t) d x \leq & \int_{0}^{\pi} b p u^{p}(x, t) d x \\
& +\int_{0}^{\pi} g^{p}\left(x, u, u_{x}\right) d x+\frac{p-1}{p} \int_{0}^{\pi} u^{p}(x, t) d x .
\end{aligned}
$$

Integrating with respect to the Lebesgue measure $d t$ over $(0, \tau)$ and applying Gronwall's inequality, it follows that

$$
\|u(\cdot, \tau)\|_{L^{p}([0, \pi])}^{p} \leq e^{\left(b p^{2}+p-1 / p\right) \tau}\left(\left\|u_{0}\right\|_{L^{p}([0, \pi])}^{p}+\left(C^{g}\right)^{p} \tau\right),
$$

which establishes the result.

5. Dependence on the initial data. In this section, we study the dependence of the solutions with respect to initial conditions. In particular, for every fixed $t>0$ and given $u_{0}$ and $v_{0}$, we obtain a ball whose radius depends explicitly on $b$ and $g$ containing the respective solutions $u$ and $v$. Furthermore, our estimate ensures that, given two initial conditions $u_{0}$ and $v_{0}$, the corresponding solutions move away from each other exponentially.

Proposition 5.1. Let $u$ and $v$ be solutions to (1.1) with initial conditions $u_{0}$ and $v_{0}$, respectively. Also, assume that Assumptions A1 and A3 hold. Then:

$$
\|u(\cdot, t)-v(\cdot, t)\|_{L^{2}([0, \pi])} \leq\left\|u_{0}-v_{0}\right\|_{L^{2}([0, \pi])} e^{\left(b+C^{g}\right) t},
$$

for every $t>0$.

Proof. Let

$$
w \doteq u-v .
$$

Notice that $w$ solves the following linear equation:

$$
\left\{\begin{array}{l}
w_{t}-w_{x x}=\left(b+g^{\prime}(z)\right) w \\
w(x, 0)=w_{0}(x)
\end{array}\right.
$$

for some $z$, where $w_{0}=u_{0}-v_{0}$. It is also easy to verify that $w$ satisfies homogeneous Neumann boundary conditions. 
Multiplying (5.1) by $w$ and integrating by parts one obtains

$$
\frac{d}{d t} \int_{0}^{\pi} w^{2}(x, t) d x \leq 2 \int_{0}^{\pi}\left(b+\left|g^{\prime}(z)\right|\right) w^{2}(x, t) d x,
$$

which in turn yields

$$
\int_{0}^{\pi} w^{2}(x, \tau) \leq 2\left(b+C^{g}\right) \int_{0}^{\tau} \int_{0}^{\pi} w^{2}(x, t) d x d t+\int_{0}^{\pi} w_{0}^{2}(x) d x .
$$

Gronwall's inequality ensures that

$$
\|w(\cdot, \tau)\|_{L^{2}([0, \pi])}^{2} \leq\left\|w_{0}\right\|_{L^{2}([0, \pi])}^{2} e^{2\left(b+C^{g}\right) t} .
$$

Because $w=u-v$, the result follows.

Corollary 5.2. Let $u$ be a solution of (1.1)-(1.2) with initial condition $u_{0}$, and assume that Assumptions $\mathrm{A} 1$ and $\mathrm{A} 3$ hold. Then

$$
\|u(\cdot, t)\|_{L^{2}([0, \pi])}^{2} \leq e^{2\left(b+C^{g}\right) t}\left\|u_{0}\right\|_{L^{2}([0, \pi])}^{2} .
$$

Proof. It is obtained in [19] the existence of a stationary solution for the equation (1.1). This implies that we can take $v(\cdot, t)$ in Proposition 5.1 to be the equilibrium $v(\cdot) \equiv 0$. Then, we obtain

$$
\|u(\cdot, t)\|_{L^{2}([0, \pi])} \leq e^{\left(b+C^{g}\right) t}\left\|u_{0}\right\|_{L^{2}([0, \pi])},
$$

which concludes the proof.

Remark 5.3. Since we can assume that $\epsilon=C^{g}$ in Proposition 4.1, the above corollary provides a better estimate for the growth of grow-up solutions to equation (1.1).

Remark 5.4. Since $X^{\alpha} \subset L^{2}$, the estimate in Corollary 5.2 can be compared to that in Section 3 for $u(\cdot, t)$ in $X^{\alpha}$.

It is also worth noting that Corollary 5.2 improves the results obtained in Corollary 4.4 and Proposition 4.5 as it yields estimates on the following: 
Corollary 5.5. Let $u$ be a solution of (1.1)-(1.2) with initial condition $u_{0}$, and assume that Assumptions A1 and A3 hold. Then:

$$
\|u(\cdot, t)\|_{W^{1,2}([0, \pi])} \leq e^{\left(b+C^{g}\right) t}\left\|u_{0}\right\|_{W^{1,2}},
$$

and, for $p>2$,

$$
\|u(\cdot, t)\|_{L^{p}([0, \pi])} \leq C e^{\left(b+C^{g}\right) t}\left\|u_{0}\right\|_{W^{1,2}([0, \pi])},
$$

for a constant $C$ which does not depend either on $t$ or $g$.

Remark 5.6. It is worth noting that the bounds obtained in Corollary 5.2 are consistent with those obtained in [13] for the trajectories on the attracting inertial manifold associated with (1.1), see Section 7.

6. Stationary problem. In this section, we study the stationary equation related to equations (1.1)-(1.2). That is to say, we are interested in the following:

$$
\left\{\begin{array}{l}
u_{x x}+b u+g\left(x, u, u_{x}\right)=0 \text { in }[0, \pi] \times(0,+\infty) \\
u_{x}(0)=u_{x}(\pi)=0 .
\end{array}\right.
$$

Proposition 6.1. Let $u$ be a solution of the stationary problem (6.1), and assume that Assumptions A1-A2 hold. Then, for any $p$ and $\alpha$ satisfying

$$
\alpha=\frac{p-2}{2 p}
$$

we have

$$
\|u\|_{L^{p}([0, \pi])} \leq C(b+1)\|u\|_{L^{2}([0, \pi])}+\widetilde{C}_{g}\|u\|_{L^{2}([0, \pi])}^{1-\alpha},
$$

where $\widetilde{C}_{g}$ is a constant depending on $g$ and $\alpha$.

Proof. Multiply equation (6.1) by $u$ and integrate by parts to obtain

$$
\int_{0}^{\pi} u_{x}^{2}(x) d x \leq b \int_{0}^{\pi} u^{2}(x) d x+\int_{0}^{\pi} g\left(x, u, u_{x}\right) u(x) d x .
$$

The Cauchy inequality with $\epsilon$ then yields

$$
\left\|u_{x}\right\|_{L^{2}([0, \pi])}^{2} \leq(b+\epsilon)\|u\|_{L^{2}([0, \pi])}^{2}+\frac{\pi}{4 \epsilon} C^{g} .
$$


It then follows from the Gagliardo-Nirenberg inequality that

$$
\begin{aligned}
\|u\|_{L^{p}([0, \pi])}^{2} & \leq C\|u\|_{L^{2}([0, \pi])}^{1-\alpha}\|u\|_{W^{1,2}([0, \pi])}^{\alpha} \\
& \leq C\|u\|_{L^{2}([0, \pi])}^{1-\alpha}\left(\|u\|_{L^{2}([0, \pi])}^{2}+(b+\epsilon)\|u\|_{L^{2}([0, \pi])}^{2}+C_{g}\right)^{\alpha / 2},
\end{aligned}
$$

where $C_{g}=\pi /(4 \epsilon) C^{g}$. By choosing an appropriate $\epsilon$ we obtain

$$
\|u\|_{L^{p}([0, \pi])}^{2} \leq C\|u\|_{L^{2}([0, \pi])}^{1-\alpha}\left((b+1)\|u\|_{L^{2}([0, \pi])}^{2}+\widetilde{C}_{g}\right)^{\alpha / 2},
$$

where $\widetilde{C}$ is a constant depending on $g$ and $\alpha$.

Next, we specialize the parameter $b$ and obtain a uniform bound for the $L^{2}$ norm of the solutions to the stationary problem.

Lemma 6.2. Let $u$ be a solution to (6.1), and assume that Assumptions A1-A2 hold. Then

$$
\|u\|_{L^{2}([0, \pi])} \leq \frac{C^{g}}{b}+\left\|u_{x}\right\|_{L^{2}([0, \pi])} .
$$

Proof. Integrate (6.1) to obtain

$$
\left|\frac{1}{\pi} \int_{0}^{\pi} u(x) d x\right| \leq \frac{C^{g}}{b} .
$$

The result then follows from the Poincaré inequality by noticing that the optimal Poincaré constant in this case is equal to 1 , see [1].

Lemma 6.3. Let $u$ be a solution (6.1), and assume that Assumptions $\mathrm{A} 1-\mathrm{A} 2$ hold. Then, for every $\varepsilon>0$,

$$
\left\|u_{x}\right\|_{L^{2}([0, \pi])}^{2} \leq(b+\varepsilon)\|u\|_{L^{2}([0, \pi])}^{2}+C_{g},
$$

where

$$
C_{g}=\frac{\pi}{4 \varepsilon}\left(C^{g}\right)^{2}
$$

Proof. Multiply (6.1) by $u$ and integrate by parts to obtain

$$
\int_{0}^{\pi}\left|u_{x}(x)\right|^{2} d x=b \int_{0}^{\pi} u^{2}(x) d x+\int_{0}^{\pi} g\left(x, u, u_{x}\right) u(x) d x .
$$


Cauchy's inequality with $\varepsilon$ yields

$$
\int_{0}^{\pi}\left|g\left(x, u, u_{x}\right) u(x)\right| d x \leq \varepsilon \int_{0}^{\pi} u^{2}(x)+\frac{\pi}{4 \varepsilon}\left(C^{g}\right)^{2} .
$$

By combining (6.4) and (6.5), the proof is completed.

Proposition 6.4. Let $u$ be a solution (6.1), and assume that Assumptions $\mathrm{A} 1-\mathrm{A} 2$ and $\mathrm{A} 4$ hold. Then,

$$
\|u\|_{L^{2}([0, \pi])} \leq C_{g, b}
$$

where $C_{g, b}>0$ depends solely on $b$ and $g$.

Proof. By combining Lemmas 6.2 and 6.3 it follows that

$$
\|u\|_{L^{2}([0, \pi])} \leq \frac{C^{g}}{b}+\frac{\sqrt{\pi} C^{g}}{2 \sqrt{\varepsilon}}+\sqrt{(b+\varepsilon)}\|u\|_{L^{2}([0, \pi])} .
$$

Because $0<b<1$, and $\varepsilon>0$ is arbitrary, absorbing $\sqrt{(b+\varepsilon)}\|u\|_{L^{2}([0, \pi])}$ on the left-hand side yields

$$
\|u\|_{L^{2}([0, \pi])} \leq \frac{1}{1-\sqrt{(b+\varepsilon)}}\left(\frac{C^{g}}{b}+\frac{\sqrt{\pi} C^{g}}{2 \sqrt{\varepsilon}}\right)
$$

which concludes the proof.

Remark 6.5. We notice that, in Proposition 6.4, it is assumed that $0<b<1$. It is important to mention that 0 and 1 are critical values of $b$, as they correspond to a degeneracy condition for equation (1.1); see Section 7.

Corollary 6.6. Let $u$ be a solution (6.1), and assume that Assumptions $\mathrm{A} 1-\mathrm{A} 2$ and $\mathrm{A} 4$ hold. Then,

$$
\|u\|_{L^{p}([0, \pi])} \leq C_{g, b, p},
$$

where $C_{g, b, p}>0$ depends solely on $g, b$ and $p$.

Proof. Corollary 6.6 follows from Propositions 6.1 and 6.4 . 
7. Concluding remarks. It is well known that solutions of (1.1)(1.2) either converge to an equilibrium or grow-up to infinity with time $[\mathbf{5}, \mathbf{1 8}]$. Therefore, in order to achieve a better understanding of the long time behavior of the solutions, it is important to obtain two distinct classes of information, namely, estimates for the growth rate of the grow-up solutions as well as uniform bounds for the equilibria.

Motivated by the this consideration, the present paper reports on three main groups of results. We obtain estimates for the growth of the solutions in $L^{p}$, for $p \geq 2$, and $W^{1,2}$ which depend on the nonlinearity $g$ and on $b$. Moreover, an upper bound for the distance between two distinct solutions is obtained in $L^{2}$. Lastly, uniform estimates for the bounded equilibria are obtained in $L^{p}$, for every $p>1$.

In the context of the existing theory, several problems related to our results are worth mentioning. In what follows, some of those are briefly discussed.

As one can observe in [5], an exponential growth for the $L^{2}$-norm of the unbounded solutions is expected. The estimate in Corollary 5.2 corroborates this fact by providing a rigorous proof.

Moreover, as mentioned in Section 3, (1.1)-(1.2) admits bounded equilibria and equilibria at infinity. On one hand, it is known that $b=n^{2}, n \in \mathbb{N}$, leads to non-hyperbolic equilibria at infinity, that is, equilibria with the eigenvalue $\lambda=0$ associated. For these critical values of $b$, bounded equilibria may accumulate on a pair of equilibria at infinity. See [5] and [15, subsection 5.3]. On the other hand, by assuming that $0<b<1$, we obtain in Proposition 6.4 a uniform bound for the $L^{2}$-norm of the equilibria to equation (1.1), which depends explicitly on $b$; as $b$ goes to 0 or 1 this estimate tends to $+\infty$. Therefore, our estimate is consistent with the expected behavior of the equilibria for the critical values $b=0,1$.

To conclude these remarks, we mention a connection between one of our estimates and a result from the theory of inertial manifolds that applies in the context of the present paper.

It is known that there exists an inertial manifold $\mathcal{M}$, that is, an invariant exponentially attracting manifold, of finite dimension $N$, associated with equation $(1.1)$, see $[\mathbf{5}, \mathbf{1 8}]$. This implies that every solution $u$ of (1.1)-(1.2) approaches $\mathcal{M}$ exponentially. In [13], a lower bound is obtained for the growth of trajectories on the inertial manifold 
$\mathcal{M}$. On the other hand, in Corollary 5.2, we have obtained an upper bound for the growth rate of solutions to (1.1). By taking $N$ sufficiently large, one can easily verify that these bounds are consistent.

Acknowledgments. The authors thank D. Gomes and two anonymous referees for their comments and suggestions on the present paper.

\section{REFERENCES}

1. G. Acosta and R.G. Durán, An optimal poincaré inequality in L1 for convex domains, Proc. Amer. Math. Soc. 132 (2004), 195-202.

2. H. Amann, Global existence for semilinear parabolic systems, J. reine angew. Math. 360 (1985), 47-83.

3. A.V. Babin and M.I. Vishik, Attractors of evolution equations, NorthHolland, Amsterdam, 1992.

4. N. Ben-Gal, Non-compact global attractors for slowly non-dissipative pdes II: The connecting orbit structure, J. Dyn. Diff. Equat., to appear.

5. , Grow-up solutions and heteroclinics to infinity for scalar parabolic PDEs, Ph.D. thesis, Brown University, Providence, RI, 2010.

6. , Non-compact global attractors for slowly non-dissipative pdes I, The asymptotics of bounded and grow-up heteroclinics, preprint, 2011.

7. H. Brezis, Functional analysis, Sobolev spaces and partial differential equations, Springer, New York, 2011.

8. P. Brunovskỳ and B. Fiedler, Connecting orbits in scalar reaction diffusion equations, Dynam. Rep. 1 (1988), 57-89.

9. __ Connecting orbits in scalar reaction diffusion equations II, The complete solution, J. Diff. Equat. 81 (1989), 106-135.

10. L.C. Evans, Adjoint and compensated compactness methods for HamiltonJacobi PDE, Arch. Rat. Mech. Anal. 197 (2010), 1053-1088.

11. B. Fiedler and C. Rocha, Heteroclinic orbits of semilinear parabolic equations, J. Diff. Equat. 125 (1996), 239-281.

12. D.A. Gomes, E. Pimentel and H. Sánchez-Morgado, Time-dependent meanfield games in the subquadratic case, Comm. Partial Diff. Equat. 40 (2015), 40-76.

13. A.Y. Goritskii and V.V. Chepyzhov, Dichotomy property of solutions of quasilinear equations in problems on inertial manifolds, Sbor. Math. 196 (2005), 485.

14. J K. Hale, Asymptotic behavior of dissipative systems, Math. Surv. Mono. 25, American Mathematical Society, Providence, RI, 1988.

15. J. Hell, Conley index at infinity, Ph.D. thesis, Freie Universität, Berlin, 2009 .

16. D. Henry, Geometric theory of semilinear parabolic equations, volume 840 , Springer-Verlag, Berlin, 1981. 
17. O. Ladyzhenskaya, Attractors for semi-groups and evolution equations, Lincei Lectures, Cambridge University Press, Cambridge, 1991.

18. J. Pimentel, Asymptotic behavior of slowly non-dissipative systems, Ph.D. thesis, Universidade de Lisboa, Lisbon, Portugal, 2014.

19. J. Pimentel and C. Rocha, A permutation related to non-compact global attractors for slowly non-dissipative equations, J. Dyn. Diff. Equat. 28 (2016), 128.

20. C. Rocha, Properties of the attractor of a scalar parabolic pde, J. Dyn. Diff. Equat. 3 (1991), 575-591.

21. F. Rothe, A priori estimates for reaction-diffusion systems, in Nonlinear diffusion equations and their equilibrium states II, Springer, New York, 1988.

Instituto Nacional de Matemática Pura e Aplicada-IMPA. Estrada Dona Castorina 110, 22460-320, Rio de Janeiro, RJ, Brazil

Email address: edgardap@impa.br

Instituto de CiÊncias Matemáticas e de ComputacÃo-ICMC-USP.

Av. Trabalhador Sancarlense 400, 13566-590, São Carlos, SP, Brazil

Email address: jpimentel@icmc.usp.br 